\title{
AÇÃO DA ALOE VERA NO REPARO TECIDUAL EM HUMANOS: UMA REVISÃO SISTEMÁTICA
}

Julliana Dias Pinheiro' Mestranda em Ciências da Saúde, Universidade Federal do Tocantins, Especialista em Terapia Intensiva, JK -DF. E-mail:

julliana.dias@hotmail.com

Guilherme Nobre L. do Nascimento $^{2}$

Laboratório de Ciências Básicas e da Saúde (LaCiBS)

Universidade Federal do

Tocantins. E-mail:

guilherme.nobre@uft.edu.br

\section{ALOE VERA ACTION IN HUMAN RESOURCES: A SYSTEMATIC REVIEW}

\section{RESUMO}

O tratamento das lesões de pele por pressão é complexo e exige a participação de uma equipe multidisciplinar, a fim de se evitar infecções locais e generalizadas, cicatrizes, desconforto e traumas psicológicos ao paciente. Em 2012, o SUS incluiu, em sua relação de medicamentos essenciais à população (RENAME), o fornecimento de um medicamento fitoterápico à base de babosa (Aloe vera), os qual possui ação cicatrizante muito conhecida na medicina popular. Este estudo trata-se de revisão sistemática que teve como objetivo geral reunir e sintetizar resultados de pesquisas que evidenciam a eficácia do fitoterápico a base de Aloe vera na cicatrização de lesões teciduais. Concluiu-se que a utilização do medicamento fitoterápico à base de Aloe vera no tratamento de lesões de pele por pressão é promissora para melhores resultados na evolução e qualidade do processo de cicatrização e reparação tecidual.

PALAVRAS-CHAVE: Aloe vera, cicatrização, feridas.

\section{ABSTRACT}

The treatment of skin lesions by pressure is complex and requires the participation of a multidisciplinary team in order to avoid local and generalized infections, scars, discomfort and psychological trauma to the patient. In 2012, SUS included, in its list of essential medicines to the population (RENAME), the supply of an herbal medicine based on aloe (Aloe vera), which has healing action well known in folk medicine. This study is a systematic review that had as general objective to gather and synthesize research results that evidence the effectiveness of the herbal remedy based on Aloe vera in the healing of tissue lesions. It was concluded that the use of the herbal medicine based on Aloe vera in the treatment of skin lesions by pressure is promising for better results in the evolution and quality of the healing process and tissue repair.

KEYWORDS: Aloe, healing, wounds. 


\section{INTRODUÇÃO}

A Aloe vera, planta conhecida popularmente como babosa, apresenta conteúdo incolor de aspecto viscoso extraído do parênquima das folhas, possui ação cicatrizante, antibacteriana, antifúngica e antiviral, seu gel é amplamente empregado no tratamento tópico de queimadura, estudos demonstram o potencial e a eficácia na cicatrização de lesões desta natureza (COLET et al., 2015). De acordo com Ramos e Pimentel (2011), o uso tópico da Aloe vera desempenha o papel de fornecer maior oxigenação ao tecido, aumentando a vascularização e a quantidade de colágeno na remodelagem do tecido cicatricial.

Segundo Vasconcelos e Caliri (2017) a reparação tecidual das lesões é um processo biológico complexo que requer intervenção clínica, envolve a organização de células, sinais químicos e remodelamento da matriz extracelular, com o objetivo de promover a restauração tecidual. A terapia para lesões tissulares busca a oclusão da lesão de forma a se alcançar a cicatriz funcional sem afetar a estética anatômica. As feridas resultam de processos multicausais, incluindo queimaduras, doenças arteriais, cirurgia, pressão local por tempo prolongado e trauma, e podem ser classificadas como aguda ou crônica.

No tratamento e reparação tecidual de feridas, o emprego de novos biomateriais e produtos médico-hospitalares de natureza diversa mostram-se relevantes, pois novas descobertas buscam meios para acelerar o processo de cicatrização, minimizando os desconfortos do paciente, facilitando a prestação dos cuidados pelas equipes multiprofissionais e otimizando o tempo de internação hospitalar e gastos com essa internação (FERREIRA; PAULA, 2013).

Estudos comprovam a eficiência de distintos produtos na cooperação do processo de reparo e cicatrização de lesões tissulares, dentre eles, pomada a base de Aloe vera que apresenta um poder emoliente e suavizante, além das vitaminas $\mathrm{C}, \mathrm{E}$, do complexo $\mathrm{B}$ e ácido fólico, contém minerais, aminoácidos essenciais e polissacarídeos que estimulam o crescimento dos tecidos e a regeneração celular (PARENTE et al., 2013).

Diante do exposto o objetivo desta revisão sistemática é descrever a ação do Aloe vera na reparação de danos tissulares.

\section{MATERIAL E MÉTODOS}

Para seleção e coleta de material bibliográfico foi realizado uma pesquisa na Biblioteca Virtual de Saúde (BVS) onde estavam indexadas as bases de dados Literatura Internacional em Ciências da Saúde (MEDLINE), Literatura LatinoAmericana e do Caribe em Ciências da Saúde (LILACS) e Base de Dados de Enfermagem (BDENF), as quais continham os artigos selecionados. Foram eleitos artigos com relevância clínica e científica, usando a combinação dos descritores Aloe vera, feridas e cicatrização; e também dos descritores Medical Subject Headings (MESH) em língua inglesa: Aloe, wounds, healing. Foram incluídos artigos originais, nos idioma português, espanhol e inglês, que dissertassem sobre a utilização de Aloe vera em feridas agudas e crônicas, em humanos. Não foi utilizado marco temporal devido possuir poucos artigos que tratavam sobre o referido tema. Os critérios utilizados para exclusão dos artigos foram: artigos primários que abordavam a aplicação de Aloe vera em animais, artigos que descreviam a morfologia da planta Aloe vera, artigos onde o objeto 
de estudo e aplicação de Aloe vera apresentava alteração genética, artigos do tipo revisão de literatura e artigos em duplicidade.

\section{RESULTADOS E DISCUSSÃO}

Nas bases de dados identificaram-se 173 publicações. A partir dos critérios de seleção, apenas 05 artigos relacionavam a utilização do Aloe vera em feridas agudas e/ou crônicas, em humanos, dos quais um foi eliminado e, dessa forma, 04 artigos foram incluídos na revisão sistemática. Os detalhes dos passos percorridos para seleção dos artigos estão descritos na figura abaixo:

Figura 1

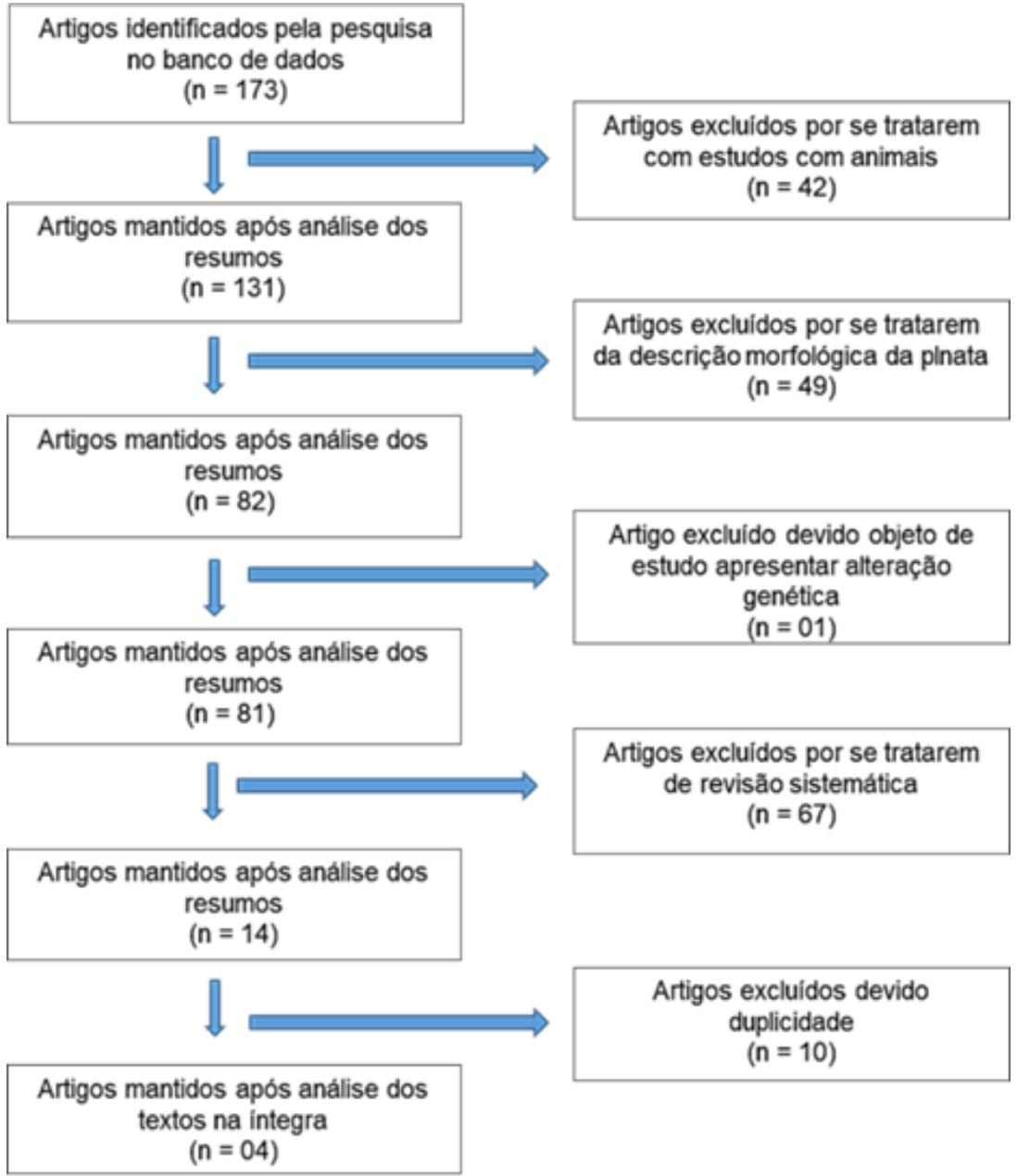

A partir do roteiro de inclusão foram transcritas as informações detalhadas de cada estudo selecionado, destacando o título do artigo, autor, ano de publicação 
e país do estudo, tipo de pesquisa, objetivos e resultados encontrados de acordo com cada tratamento adotado, relatados no quadro abaixo.

Quadro I: Distribuição das referências bibliográficas relacionados ao uso de Aloe vera no tratamento e restauração tissular em feridas, segundo o título, autor, ano de publicação e país do estudo, tipo de pesquisa, objetivos e resultados.

Quadro I: Distribuição das referências bibliográficas relacionados ao uso de Aloe vera no tratamento e restauração tissular em feridas, segundo o título, autor, ano de publicaçäo e país do estudo, tipo de pesquisa, objetivos e resultados.

\begin{tabular}{|c|c|c|c|c|}
\hline Titulo & $\begin{array}{l}\text { Autor, ano de } \\
\text { publicaçăo/ País } \\
\text { do estudo }\end{array}$ & $\begin{array}{l}\text { Tipo de } \\
\text { Pesquisa }\end{array}$ & Objetivos & Resultados \\
\hline $\begin{array}{l}\text { Analise do processso } \\
\text { de cicatrizaçâo de } \\
\text { feridas crônicas } \\
\text { utilizando gel de Aloe } \\
\text { arborescens e } \\
\text { Stryphnodendron } \\
\text { adstringens }\end{array}$ & $\begin{array}{l}\text { Pedrassini; } \\
\text { Tashima, 2015 / } \\
\text { Brasil }\end{array}$ & $\begin{array}{l}\text { Estudo } \\
\text { descritivo } \\
\text { de } \\
\text { abordagem } \\
\text { quali- } \\
\text { quantitativa } \\
\text { longitudinal }\end{array}$ & $\begin{array}{l}\text { Avaliar as } \\
\text { propriedades } \\
\text { cicatrizantes } \\
\text { da utilizaçâo } \\
\text { tópica de Aloe } \\
\text { arborescense } \\
\text { S } \\
\text { Stryphnoden } \\
\text { dron } \\
\text { adstringense } \\
\text { m feridas } \\
\text { crônicas. }\end{array}$ & $\begin{array}{l}\text { Barbatimáo } \\
\text { apresentou } \\
\text { propriedades } \\
\text { cicatrizantes mais } \\
\text { eficazes para } \\
\text { feridas que o Aloe } \\
\text { vera. }\end{array}$ \\
\hline $\begin{array}{l}\text { Uso de cobertura } \\
\text { com colágeno e Aloe } \\
\text { vera no tratamento } \\
\text { de ferida isquémica: } \\
\text { estudo de caso }\end{array}$ & $\begin{array}{l}\text { Dos Santos } \\
\text { Oliveira et al, } \\
2010 / \text { Brasil }\end{array}$ & $\begin{array}{l}\text { Pesquisa } \\
\text { experiment } \\
\text { al }\end{array}$ & $\begin{array}{l}\text { Desenvolvim } \\
\text { ento de novas } \\
\text { tecnologias } \\
\text { para o } \\
\text { tratamento de } \\
\text { feridas. } \\
\end{array}$ & $\begin{array}{l}\text { Boa tolerabilidade } \\
\text { e eficácia } \\
\text { terapéutica para } \\
\text { este caso em } \\
\text { particular. }\end{array}$ \\
\hline $\begin{array}{l}\text { Actividad } \\
\text { antiinflamatoria y } \\
\text { cicatrizantedel } \\
\text { ungūento rectal de } \\
\text { Aloe vera } L \text { (sábila) }\end{array}$ & $\begin{array}{l}\text { León Sarabia et } \\
\text { al, } 1999 \text { / Cuba }\end{array}$ & $\begin{array}{l}\text { Pesquisa } \\
\text { experiment } \\
\text { al. }\end{array}$ & $\begin{array}{l}\text { Avaliar a } \\
\text { atividade anti- } \\
\text { inflamatónia e } \\
\text { curativa de } \\
\text { aloe no } \\
\text { tratamento de } \\
\text { tais } \\
\text { entidades. }\end{array}$ & $\begin{array}{l}\text { A açáo } \\
\text { antiinflamatória } \\
\text { do Aloe no surto } \\
\text { hemorróidal foi } \\
\text { demonstrada } \\
\text { agudo, bem } \\
\text { como, curador e } \\
\text { resolutivo na } \\
\text { fissura anal. }\end{array}$ \\
\hline $\begin{array}{l}\text { Comparative trial of } \\
\text { Aloe vera/olive oil } \\
\text { combination cream } \\
\text { versus phenytoin } \\
\text { ream in the treatment } \\
\text { of chronic wounds }\end{array}$ & $\begin{array}{l}\text { Panahi et al, } \\
2015 \text { / EUA }\end{array}$ & $\begin{array}{l}\text { Estudo } \\
\text { randomizad } \\
\quad \text { duplo- } \\
\text { cego, } \\
\text { controlado. }\end{array}$ & $\begin{array}{l}\text { Avaliar o } \\
\text { efeito do Aloe } \\
\text { vera-azeite } \\
\text { (AVO) } \\
\text { creme } \\
\text { combinado no } \\
\text { processo de } \\
\text { cicatrização } \\
\text { de feridas } \\
\text { crônicas. }\end{array}$ & $\begin{array}{l}\text { O creme AVO } \\
\text { acelera } \\
\text { significativamente } \\
\text { a cura biológica } \\
\text { de feridas } \\
\text { crônicas e ajuda } \\
\text { reduzindo a } \\
\text { gravidade da dor } \\
\text { com maior } \\
\text { eficácia em } \\
\text { comparação com } \\
\text { o creme de } \\
\text { fenitoína. }\end{array}$ \\
\hline
\end{tabular}


O aproveitamento de plantas medicinais tem como pilar a tradição familiar e tornou-se realidade na medicina popular, sendo considerada uma terapia alternativa ou complementar para a promoção e recuperação da saúde (LOYA et al., 2009). As referências terapêuticas das plantas medicinais é, habitualmente, uma característica da população mais idosa, sendo que estas plantas são aproveitadas principalmente para o tratamento de menores morbidades (BALBINOT; VELASQUEZ; DÜSMAN, 2013).

Aloe vera, denominada cientificamente como Aloe vera (L.) Burm.f, Aloe barbadensis Mill., Aloe barbadensis var. chinensis Haw., Aloe perfoliata var. vera L., Aloe chinensis Bak. e Aloe vera var. chinensis Berger, está na família Liliaceae, que é uma planta tropical facilmente cultivada em climas quentes e secos, incluindo o Brasil, sendo popularmente conhecida por Babosa (LORENZI; MATOS, 2008; PALHARIN et al., 2008). Encontramos relatos de sua utilização desde a era romana, os diversos produtos cosméticos e medicamentos são extraídos do tecido mucilaginoso situado no centro da folha da planta (AKHOONDINASAB; AKHOONDINASAB; SABERI, 2014).

No ano de 2012, o Sistema Único de Saúde (SUS), incluiu em sua Relação Nacional de Medicamentos Essenciais (RENAME) doze fitoterápicos, entre eles o creme a base de babosa (Aloe vera (L.) Burm. F.) para o tratamento tópico de queimaduras de primeiro e segundo grau e como coadjuvante nos casos de psoríase (BRASIL, 2012).

Composta predominantemente por água, em torno de 99,5\%, foram identificadas nas folhas da Aloe vera diversas outras substâncias, entre elas: aminoácidos, antraquinonas, aloínas, aloeferon, esteroide, enzimas, flavonóides, glicoproteínas, taninos, saponinas, proteínas, vitaminas, minerais, polissacarídeos, potássio, manganês e sódio (DIAS et al., 2016; FREITAS et al., 2014). Estudos identificaram algumas substâncias como sendo responsáveis pelas atividades de reparação tecidual, anti-inflamatória e cicatrizante da Aloe vera, das quais foram citadas: antraquinônica, as aloínas e aloeferon (FREITAS et al., 2014; PEREIRA et al., 2014).

A cicatrização de lesões por queimadura é uma das principais indicações do gel de Aloe vera, sendo utilizada em muitos países (MAENTHAISONG, 2007). Ensaios clínicos investigando o efeito de Aloe Vera em lesões por queimadura atestou que a mesma diminuiui significativamente o tempo de cicatrização da ferida (em torno de oito dias) em comparação com 0 controle (AKHOONDINASAB; AKHOONDINASAB; SABERI, 2014). Segundo Hosseinimehr et al. (2010) essa melhora das lesões tratadas com a Aloe vera se dá devido seus potenciais efeitos antimicrobianos e anti-inflamatórios.

A medicina progride e com ela observamos o crescente interesse pela fitoterapia e pela homeopatia como opções terapêuticas (PALHARIN et al., 2008). Além dos efeitos benéficos e dos potenciais terapêuticos a fitoterapia tem se mostrado economicamente acessível (RODRíGUEZ DOMíNGUEZ et al., 2006). No Reino Unido os custos associados ao tratamento de úlceras foram estimados em 5.200 libras por ano, por doente, e nos EUA, em 7 bilhões de dólares em 2007, só em materiais e que podem crescer até 20 bilhões de dólares se forem contabilizados outros custos como: remuneração dos profissionais de saúde, hospitalização, controle da infecção, entre outros (GASPAR et al., 2010).

O estudo de Pedrassini \& Tashima, (2015) concluiu que após 14 dias de uso diário de gel a base de Aloe vera $3 \%$, nota-se a diminuição da área da ferida.

Para Dos Santos Oliveira et al. (2010), embora tenha obtido sucesso com a aplicação do produto tendo na sua composição Extrato de Aloe vera em gel $(1,5 \mathrm{ml})$, o resultado não é suficiente para afirmar a eficácia do mesmo, nem tão 
pouco para generalizações, pois faz-se necessário uma amostra mais ampliada para assim evidenciar melhores esclarecimentos acerca da utilização do produto em variados tipos de lesões, bem como dos seus efeitos sobre a reparação e cicatrização epitelial e de possíveis incômodos, vulnerabilidades e reações adversas ao paciente, embora nenhuma complicação ou incomodidade tenha sido destacado.

Segundo León Sarabia et al. (1999), afirma que um estudo feito com 48 pacientes tratados diariamente com a pomada a base de Aloe vera, destes, 44 pacientes apresentaram cicatrização das lesões ocasionadas por crise hemorroidária aguda $(91,7 \%)$ em até 14 dias de terapia, sendo notado diminuição do edema e melhora na ação anti-inflamatória, não sendo observado melhora significatica quando ao poder analgésico.

Para Panahi (2015) em seu estudo ficou evidenciado que após 30

dias de tratamento com a pomada a base de Aloe vera, noto-se melhoras significativas no tamanho da ferida, profundidade da ferida, bordas da ferida, tecido necrótico

tipo e quantidade, tipo e quantidade de exsudado, a cor do arredondamento da ferida e tecido periférico, favorecendo assim a cicatrização das lesões.

\section{CONSIDERAÇÕES FINAIS}

A utilização do medicamento fitoterápico à base de Aloe vera no tratamento de lesões de pele por pressão é auspicioso para melhores resultados na evolução e qualidade do processo de cicatrização e reparação tecidual, visto que, os estudos in vitro têm demonstrado que a Aloe vera é uma planta promissora, inclusive para bactérias multirresistentes, gerando a necessidade de mais estudos nesta temática.

\section{REFERÊNCIAS}

AKHOONDINASAB, Mohammad Reza; AKHOONDINASAB, Motahhare; SABERI, Mohsen. Comparison of healing effect of aloe vera extract and silver sulfadiazine in burn injuries in experimental rat model. World journal of plastic surgery, v. 3, n. 1, p. 29, 2014.

BALBINOT, S.; VELASQUEZ, P. G.; DÜSMAN, E. Reconhecimento e uso de plantas medicinais pelos idosos do Município de Marmeleiro-Paraná. Revista Brasileira de Plantas Medicinais, v. 15, n. 4, p. 632-638, 2013.

Brasil. Ministério da Saúde. Relação Nacional de Medicamentos Essenciais. Brasília: MS; 2012.

COLET, Christiane et al. Uso de Aloe sp. no Município de Pejuçara-RS.

Journal of Health Sciences, v. 17, n. 2, 2015.

FERREIRA, Francis Villegas; PAULA, Larissa Barbosa. Sulfadiazina de prata versus medicamentos fitoterápicos: estudo comparativo dos efeitos no 
tratamento de queimaduras. Revista Brasileira de Queimaduras, v. 12, n. 3, p. 132-9, 2013.

FREITAS, V. S.; RODRIGUES, R. A. F.; GASPI, F. O. G. Propriedades farmacológicas da Aloe vera (L.) Burm. f. Rev Bras Plantas Med, v. 16, n. 2, p. 299-307, 2014.

DIAS, Julliany Lopes et al. Avaliação in vitro da atividade antimicrobiana e do potencial citotóxico do gel de Aloe Vera: uma discussão sobre o uso em queimaduras. 2016.

DOS SANTOS OLIVEIRA, Simone Helena; SOARES, Maria Julia Guimarães Oliveira; DE SOUSA ROCHA, Pascalle. Uso de cobertura com colágeno e aloe vera no tratamento de ferida isquêmica: estudo de caso. Revista da Escola de Enfermagem da USP, v. 44, n. 2, p. 346-351, 2010.

GASPAR, Pedro João Soares et al. Impacto da formação profissional contínua nos custos do tratamento das feridas crónicas. Revista de Enfermagem Referência, n. 1, p. 53-62, 2010.

LEÓN SARABIA, Jorge Enrique et al. Actividad antiinflamatoria y cicatrizante del ungüento rectal de Aloe Vera L.(sábila). Revista Cubana de Plantas Medicinales, v. 4, n. 3, p. 106-109, 1999.

LORENZI, H.; MATOS, F. J. A. Plantas medicinais no Brasil - Nativas e exóticas. 2.ed. São Paulo: Instituto Plantarum, 2008; 244p

LOYA, Amanda M.; GONZÁLEZ-STUART, Armando; RIVERA, José O. Prevalence of polypharmacy, polyherbacy, nutritional supplement use and potential product interactions among older adults living on the United States-Mexico border. Drugs \& aging, v. 26, n. 5, p. 423-436, 2009.

MAENTHAISONG, Ratree et al. The efficacy of aloe vera used for burn wound healing: a systematic review. burns, v. 33, n. 6, p. 713-718, 2007.

PALHARIN, L.H.D.C.; NETO, E.F.; LOPES, M.P.C.; ASCÊNCIO, F.; BOSQUÊ, G.G. Efeitos fitoterápicos e homeopáticos da babosa. Revista Científica Eletrônica de Agronomia, v. VII, n.14, 2008.

PARENTE, Leila Maria Leal et al. Aloe vera: características botânicas, fitoquímicas e terapêuticas. Arte Méd Ampl, v. 33, n. 4, p. 160-4, 2013.

PANAHI, Y. et al. Comparative trial of Aloe vera/olive oil combination cream versus phenytoin cream in the treatment of chronic wounds. Journal of wound care, v. 24, n. 10, p. 459-465, 2015.

PEDRASSINI, E. H. C.; TASHIMA, C. M. Análise do processo de cicatrização de feridas crônicas utilizando gel de Aloe arborescens e Stryphnodendron adstringens. V Jornada de Iniciação Científica da UENP, 2015. 
PEREIRA, Gabriela Garrastazu et al. Polymeric films loaded with vitamin E and Aloe vera for topical application in the treatment of burn wounds. BioMed research international, v. 2014, 2014.

RAMOS, A. de P.; PIMENTEL, L. C. Ação da Babosa no reparo tecidual e cicatrização. Brazilian Journal of Health, v. 2, n. 1, p. 40-48, 2011.

RODRÍGUEZ DOMÍNGUEZ, lleana et al. Beneficios del Aloe Vera I.(sábila) en las afecciones de la piel. Revista Cubana de Enfermería, v. 22, n. 3, p. 0-0, 2006.

VASCONCELOS, Josilene de Melo Buriti; CALIRI, Maria Helena Larcher. Ações de enfermagem antes e após um protocolo de prevenção de lesões por pressão em terapia intensiva. Escola Anna Nery, v. 21, n. 1, 2017.

Recebido em: 14-03-2019

Aceito em: 11-11-2019 